\title{
Punzones de madera arqueológicos de Punta Medanosa (costa norte de Santa Cruz, Argentina)
}

\section{Laura Ciampagna}

\section{Resumen}

Se presenta el estudio de dos punzones de madera hallados en las proximidades de Roca del Cura, localidad arqueológica de Punta Medanosa, Santa Cruz, Argentina. El objetivo de este trabajo es describir la morfología de los instrumentos e identificar taxonómicamente la madera de estos punzones. Se discute la funcionalidad de los artefactos al considerar el soporte con el que fueron realizados en relación con otros instrumentos en hueso hallados en la costa norte de Santa Cruz (CNSC) y trabajos realizados por otros investigadores sobre cadenas operativas de cuero y cestería. Los artefactos se observaron bajo lupa binocular, se realizaron los cortes histológicos diagnósticos de la madera, se observaron al microscopio óptico y se comparó con material de referencia y bibliografía específica. La madera identificada pertenece a Schinus sp. (molle), arbustos que se encuentran en la actualidad en la zona circundante al área del hallazgo. Se sugiere la manufactura local de los instrumentos y su posible utilización en el trabajo de materiales blandos como fibras para cestería y decoración incisa en cerámica.

\section{Archaeological wooden awls from Punta Medanosa (North Coast of Santa Cruz, Argentina)}

\begin{abstract}
In this article, we present the study of two wooden awls found near the archaeological site of Roca del Cura in the archaeological area of Punta Medanosa (Santa Cruz, Argentina). Our aim was to identify the wooden taxa, as well as provide a morphological description of the tools. The artifacts functionality is discussed in relation to other bone instruments found along the Northern Santa Cruz Coast (CNSC), including research on leather and basketry operating chains, undertaken by other researchers. The artifacts were examined under a binocular microscope. While histological sections of the wood were taken and compared against reference material and specialized bibliography. The wood was identified as Schinus sp. (molle); a shrub taxa that is still present in the surrounding area. We suggested the local manufacture of these tools, and that they may have been employed on soft materials such as fibers for basket weaving and ceramic decoration.
\end{abstract}

* División Arqueología, Facultad de Ciencias Naturales y Museo, Universidad Nacional de La Plata (UNLP) -

CONICET. Paseo del Bosque s/n (CP B19ooFWA) La Plata, Buenos Aires, Argentina. E-mail: mlciampagna@gmail.com
Recibido:

30 de marzo de 2017

Aceptado:

18 de agosto de 2017

\section{Palabras claves}

Punzones

Madera

Cazadores-recolectores

Costa norte de Santa Cruz

Keywords

Awls

Wood

Hunter-gatherers

North Coast of Santa Cruz 
1. Se realizaron cortes histológicos sobre dos de los tres instrumentos para que uno, que se exhibe en el Museo local de la Estancia El Amanecer, no presentara marcas de los cortes anatómicos.

\section{Introducción}

En este trabajo se presenta el análisis tecno-morfológico y anatómico de dos punzones de madera recuperados en superficie asociados a sitios concheros próximos al afloramiento de Roca del Cura, en la localidad arqueológica Punta Medanosa (Figuras 1 y 2), sector sur de la ría Deseado. Estos instrumentos forman parte de la colección privada de la Estancia El Amanecer (EA), en la cual también se encuentra otro punzón de madera que no fue analizado en este trabajo ${ }^{1}$ y cinco puntas de arpón. Esta colección está compuesta también por material lítico (i.e. láminas, denticulados, rompecráneos, entre otros), cerámica, ocre y valva (i.e. cuenta de collar) recuperados en superficie en distintos sitios de la localidad arqueológica de Punta Medanosa asignados cronológicamente al Holoceno tardío (Hammond, Zilio y Zubimendi, 2016). En el sitio Cueva del Negro, también ubicado en el sector sur de la ría Deseado y a $20 \mathrm{~km}$ en línea recta de Punta Medanosa, se recuperó en estratigrafía un fragmento de arpón manufacturado con madera de Berberis sp. L. (Capparelli, Castro y Ciampagna, 2009). Hasta el momento, los punzones de madera y el fragmento de arpón son los únicos artefactos realizados sobre leños recuperados en la Costa Norte de Santa Cruz (CNSC). En el sitio Cueva del Negro se hallaron, además, tres piezas que fueron definidas como punzones, leznas y agujas en soporte de hueso de aves marinas y seis puntas de arpón monodentadas de espaldón simple pertenecientes a puntas de arpón móvil sobre elementos óseos de cetáceos y pinnípedos (Beretta y Zubimendi, 2017; Beretta, Zubimendi, Ciampagna, Ambrústolo y Castro, 2013b). El estudio de instrumentos de morfología similar en soporte madera y óseo de la colección de la Estancia El Amanecer y el sitio Cueva del Negro permitirían abordar la diversidad de materias primas para un mismo sistema tecnológico desarrollado por los grupos cazadores recolectores que ocuparon la CNSC en el Holoceno tardío.

En este sentido, el objetivo general de este trabajo es describir la morfología de los instrumentos e identificar taxonómicamente la madera de los punzones de la colección Estancia El Amanecer y abordar su potencial funcionalidad en comparación con instrumentos similares realizados en hueso que provienen de la CNSC. Se proponen los siguientes objetivos específicos del material de estudio: realizar los cortes histológicos diagnósticos para la determinación taxonómica de los punzones de madera de la colección EA y registrar las dimensiones de los punzones de madera de la colección EA y la presencia de marcas de procesamiento (i.e. pulido). Y a partir de antecedentes bibliográficos se apunta a: recopilar instrumentos en madera aguzados Patagonia continental Argentina y los potenciales usos asignados y caracterizar los punzones manufacturados en soporte óseo del sitio Cueva del Negro y su posible funcionalidad.

Los objetivos, general y específicos, se formularon en función de contrastar dos hipótesis:

» Hipótesis 1: Los punzones de madera de la colección EA son similares en morfología a los punzones óseos del sitio Cueva del Negro.

》Hipótesis 2: Los punzones de madera de la colección EA fueron utilizados por grupos cazadores recolectores en alguna etapa de las cadenas operativas de cestería, cuero y cerámica durante el Holoceno tardío.

\section{Localidad arqueológica Punta Medanosa}

El accidente geográfico de la punta Medanosa se extiende desde el sur de Isla Lobos hasta el final de la Punta en el litoral Atlántico (Figura 1). La punta mide $12 \mathrm{~km}$ de largo por $6 \mathrm{~km}$ de ancho y se encuentra separada del continente por un sistema de marismas (Ensenada Ferrer). En el norte está conformada por la terraza marina, cubierta por dunas y depósitos de arena. El afloramiento Roca del Cura se encuentra en esta área 


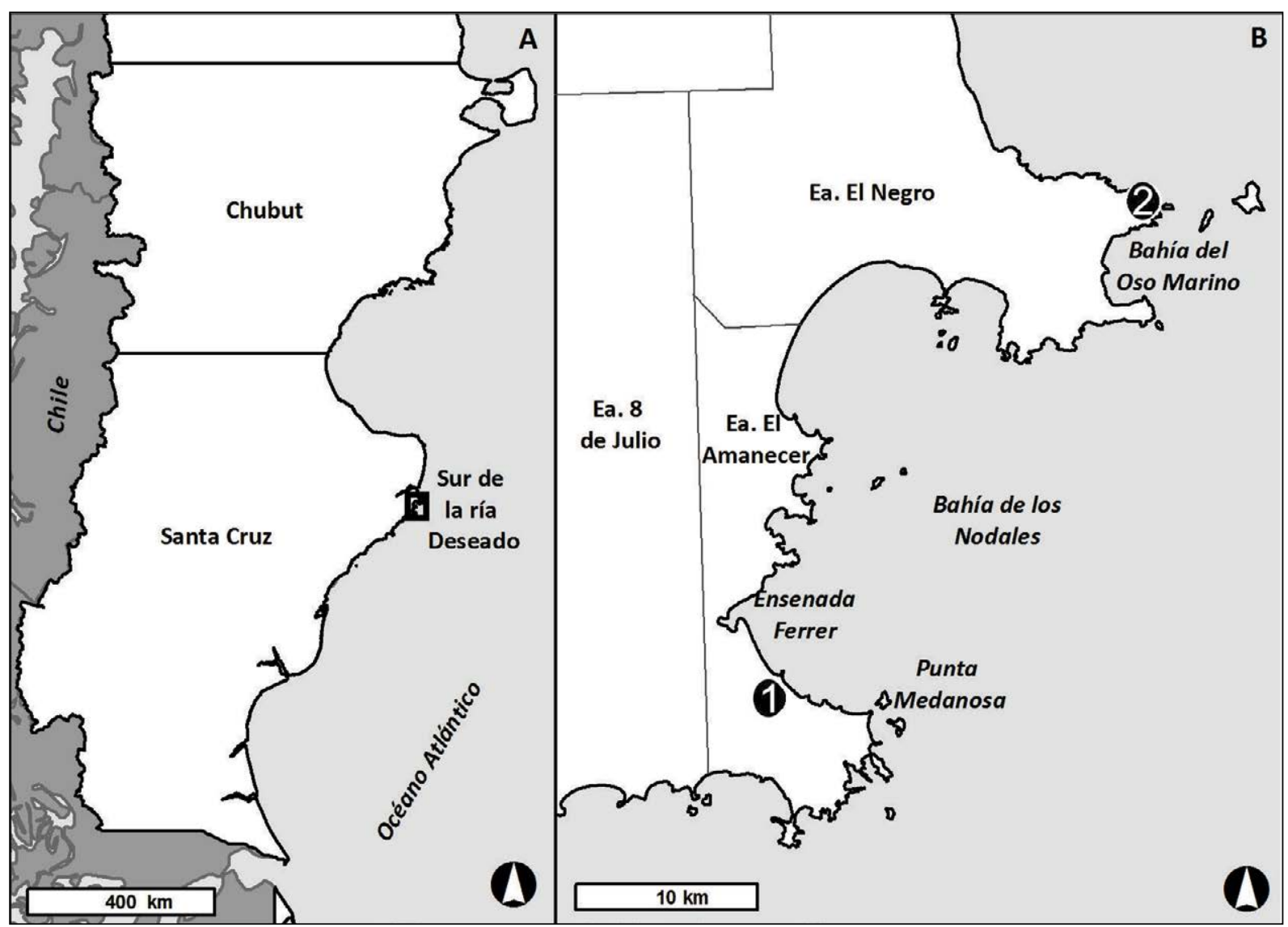

Figura 1. Ubicación del sector sur de la ría Deseado de la CNSC donde se localizaron los punzones de madera y óseos. A: Ubicación del sector sur de la ría Deseado; B: 1 Afloramiento Roca del Cura en la localidad arqueológica de Punta Medanosa; 2 Sitio Cueva del Negro.

sobre el fondo de la Ensenada Ferrer (Figura 1, Figura 3A). Se hallaron sitios concheros estratificados que apoyan sobre sedimentos de origen marino (Figura 3B). En las costas este y sur se observa el afloramiento de rocas ignimbríticas del grupo Bahía Laura (Castro, Zubimendi y Ambrústolo, 2011; Hammond, 2013).

El clima es templado frío, árido de meseta y en relación con la vegetación se registra el límite sur de la estepa arbustiva del golfo San Jorge para pasar a predominar la estepa de arbustos enanos (Oliva, González y Rial, 2001). Se destacan por su ubicuidad en el paisaje y mayor altura que en otros sectores de la CNSC, los arbustos de Schinus sp. L. (molle) cuya madera es semidura a semipesada según estudios previos de la autora y presenta fibras cortas (Figura 3C) (Ciampagna, 2015, 2016; Guerra et al., 2012). En cuanto a los recursos faunísticos actualmente se encuentra una colonia de reproducción de pingüinos de Magallanes (Spheniscus magallanicus), amplios bancos de moluscos y en el pasado se registraron grandes loberías en la punta e islotes adyacentes a la playa (Castro, Moreno, Andolfo y Zubimendi, 2001; Hammond, Zubimendi y Zilio, 2013; Schiavini, Yorio, Gandini, Raja Rey y De Boerma, 2005). Si bien no se han realizado estudios palinológicos en el área de estudio, se tuvo como referencia los análisis realizados por De Porras (2010) para la meseta central. En éstos se concluye que durante los 50003750 años AP se habría desarrollado y establecido una comunidad arbustiva-subarbustiva dominada por la familia Asteraceae subfamilia Asteroidea, con una gran diversidad de arbustos altos similar a la vegetación actual (De Porras, 2010, p. 113).

Los punzones de madera que se estudian en este trabajo fueron hallados en superficie por los dueños de la Estancia El Amanecer en asociación a sitios concheros próximos 


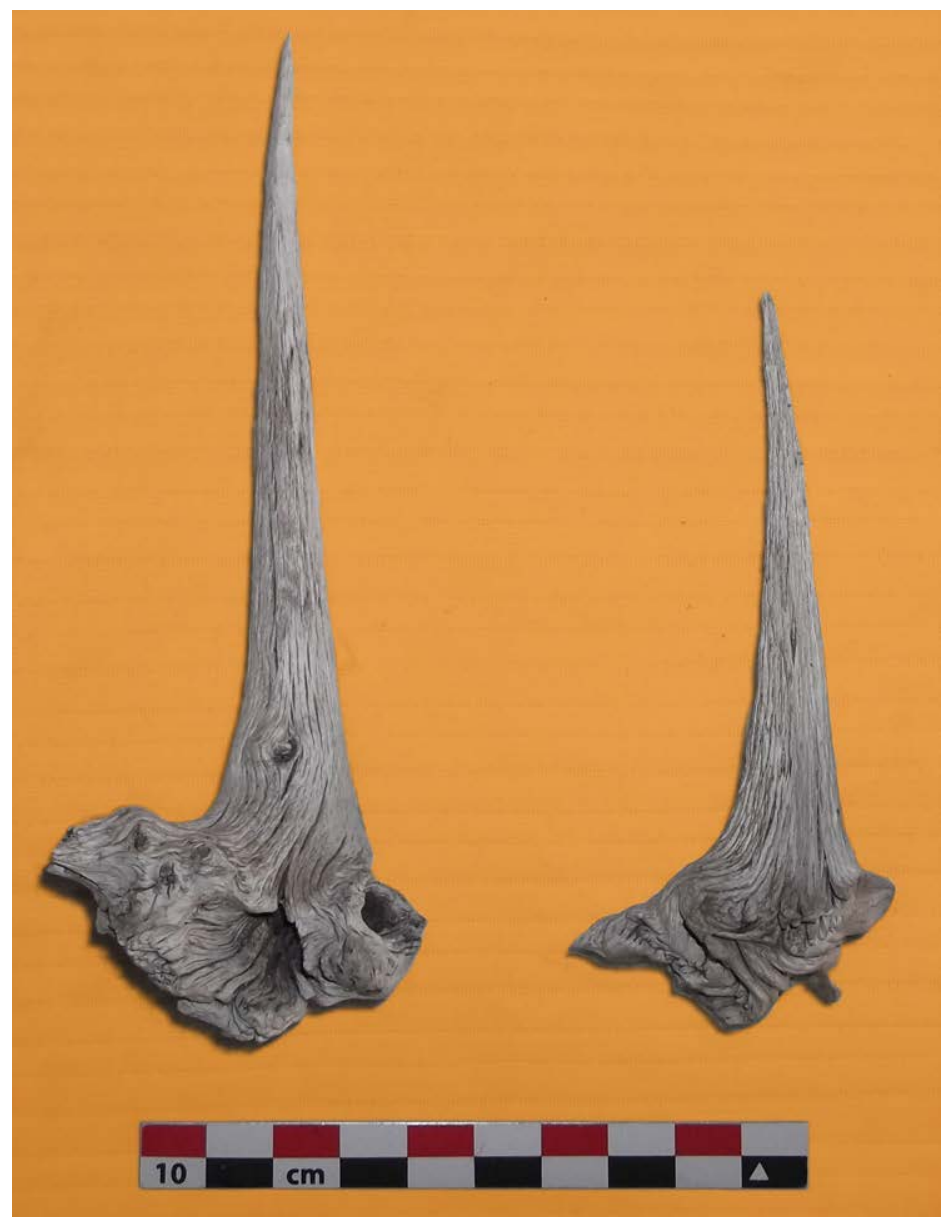

Figura 2. Punzones arqueológicos de madera.

al afloramiento de Roca del Cura, en esta localidad arqueológica. Si bien, debido a las características del hallazgo no se obtuvo mayor información contextual, se han realizado estudios arqueológicos por parte del equipo de CNSC en la localidad de Punta Medanosa y próxima al lugar donde se encontraron los instrumentos. Se han registrado sitios concheros de formas cónicas de planta subcircular emplazados en médanos, con material lítico en superficie (Castro et al., 2001). En otros sectores de la localidad de Punta Medanosa se han realizado fechados en sitios concheros que permiten afirmar la ocupación por parte de sociedades cazadoras recolectoras desde el Holoceno medio al tardío (Hammond et al., 2013; Zubimendi, Ambrústolo, Zilio y Castro, 2015). La colección Estancia El Amanecer proviene del material de superficie de los sitios concheros que se hallan en esta localidad arqueológica, abarcando como se mencionó en la sección Introducción material lítico, óseo, cerámico, leñoso y malacológico asignados al Holoceno tardío.

\section{Antecedentes}

\section{Instrumentos arqueológicos leñosos recuperados en CNSC y artefactos de morfología ahusada, recuperados en sitios arqueológicos de Patagonia continental Argentina}

En la CNSC, además de los instrumentos que se estudian en este trabajo, sólo se recuperó un fragmento de arpón manufacturado con madera de Berberis sp. en el sitio Cueva del Negro (Capparelli et al., 2009). Este instrumento se hallaba asociado a 


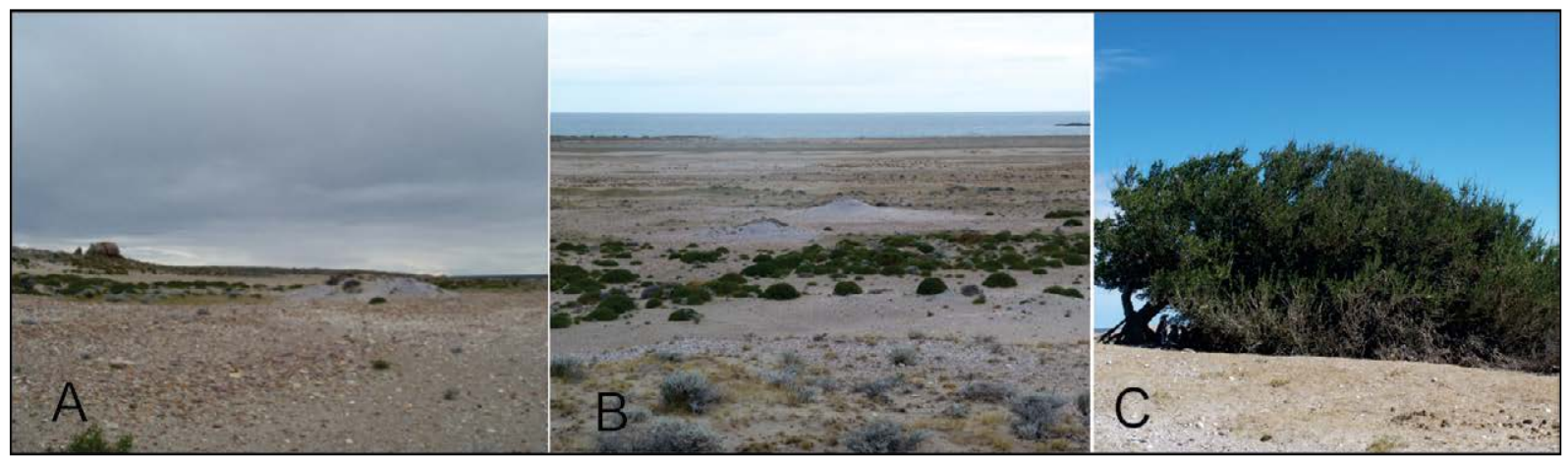

Figura 3. Ambiente de Punta Medanosa. A: Afloramiento Roca del Cura y sitios concheros. B: sitio conchero. C: Arbusto de Schinus sp. de gran porte asociado a nido de pingüinos.

seis puntas de arpón enteras y fragmentadas de soporte óseo en estratigrafía (Beretta et al., 2013b).

En una escala espacial más amplia denominada Patagonia continental argentina (PCA) existen antecedentes de artefactos en madera de morfología ahusada y en algunos casos mencionados como punzones. El área PCA es un recorte espacial arbitrario que abarca en sentido oeste-este desde la cordillera de los Andes a la costa Atlántica y en sentido Norte-Sur desde el río Colorado hasta el límite sur de la provincia de Santa Cruz. En el sitio El Manzano, en la provincia de Mendoza, se recuperó un punzón elaborado con el mango de Phragmites australis y una espina sujetada al mismo con tendones (Andreoni, 2014; Llano, 2010; Llano y Andreoni, 2012) (Tabla 1). Llano (2010) considera que este instrumente podría haber sido utilizado para perforar materiales blandos como el cuero. En el sitio Campo Moncada II se recuperó una espina de Prosopis sp. L. y una aguja de madera también elaborada con el mismo taxa (Pérez de Micou, Bellelli y Aschero, 1992). De acuerdo a Marchione y Bellelli (2013) las agujas podrían haber sido utilizadas para la costura del cuero en el sitio Campo Moncada II. En el sitio Campo Nassif I también se recuperó una aguja realizada con una espina de Schinus sp. L. (Pérez de Micou, López Campeni y Lessa Costa, 2014). Entre otros instrumentos con morfología ahusada se encuentran varillas asignadas a estacas y posibles usos de hilar (Tabla 1).

\section{Punzones de hueso en el sitio Cueva del Negro}

En el trabajo de Beretta y Zubimendi (2017) se presenta un conjunto de nueve piezas confeccionadas en hueso que abarcan punzones, leznas y/o agujas, tubo sorbedor y cuentas que provienen del sitio Cueva del Negro. Asimismo, realizaron una exhaustiva recopilación de la presencia de estos instrumentos para Patagonia argentina y chilena. El sitio Cueva del Negro se encuentra fechado por ${ }^{14} \mathrm{C}$, sobre hueso de choique y pinnípedo, entre $1170 \pm 110$ y $1730 \pm 80$ años AP (Beretta y Zubimendi, 2017; Zubimendi et al., 2011). El punzón se realizó sobre una ulna izquierda de Phalacrocorax sp. (cormorán), que conserva la epífisis proximal y presenta una punta distal aguzada obtenida a partir de una fractura oblicua en la porción distal de la diáfisis (Figura 4A) (Beretta y Zubimendi, 2017). Las dimensiones son $126,9 \mathrm{~mm}$ de longitud máxima, $6,02-1,25 \mathrm{~mm}$ de ancho máximo y mínimo y 7,32-1,19 mm de altura máxima y mínima. Para los autores, esta pieza podría haber sido utilizada en la manufactura de cestos, como pasantes de materiales blandos o tejidos vegetales debido a las características de tamaño, diámetro de la punta y escasa resistencia a la compresión (Beretta y Zubimendi, 2017). Otras dos piezas definidas como agujas o leznas, presentan una sección circular pero con un extremo muy aguzado y punzante de menos de $1 \mathrm{~mm}$. Una de ellas se realizó sobre la diáfisis del húmero de un ave voladora como Diomedeidae (albatros) o Procellariidae 


\begin{tabular}{|c|c|c|c|c|c|c|}
\hline Sitio & Cronología & $\mathbf{N}$ & Tecnofactura & Huellas tecnológicas & Taxa & Citas \\
\hline \multirow{5}{*}{ El Manzano } & \multirow{5}{*}{ Holoceno tardío } & 3 & $\begin{array}{l}\text { posibles usos de } \\
\text { hilar }\end{array}$ & $\begin{array}{l}\text { ahusamiento de los } \\
\text { ápices, descortezado } \\
\text { tallado y pulido }\end{array}$ & Larrea nitida & \multirow{3}{*}{$\begin{array}{l}\text { Andreoni, 2014; } \\
\text { Llano, 2010; } \\
\text { Llano y Andreoni, } \\
2012\end{array}$} \\
\hline & & 4 & espinas & una con decoración & Prosopis alpataco & \\
\hline & & 1 & $\begin{array}{l}\text { punzón (mango } \\
\text { con espina) }\end{array}$ & $s / d$ & $\begin{array}{l}\text { Phragmites } \\
\text { australis (mango) }\end{array}$ & \\
\hline & & 2 & posibles estacas & $\begin{array}{l}\text { termo-alteración, } \\
\text { descortezado pulido, } \\
\text { aguzado, pintado }\end{array}$ & $\begin{array}{l}\text { Larrea cuneifolial } \\
\text { divaricata }\end{array}$ & \multirow{2}{*}{ Andreoni, 2014} \\
\hline & & 1 & posible estaca & $s / d$ & $\begin{array}{l}\text { Tessaria } \\
\text { dodoneifolia }\end{array}$ & \\
\hline \multirow{2}{*}{ Chenque Haichol } & \multirow{2}{*}{ Holoceno tardío } & 1 & espina & $\begin{array}{l}\text { signos de utilización } \\
\text { en el extremo distal }\end{array}$ & $\mathrm{s} / \mathrm{d}$ & \multirow{2}{*}{$\begin{array}{l}\text { Ancibor, 1988- } \\
1990\end{array}$} \\
\hline & & 1 & varilla rígida & $\begin{array}{l}\text { superficie pulida y } \\
\text { extremo aguzado }\end{array}$ & $\mathrm{s} / \mathrm{d}$ & \\
\hline \multirow{2}{*}{ Campo Moncada II } & \multirow{2}{*}{ Holoceno tardío } & 1 & espina & $\mathrm{s} / \mathrm{d}$ & Prosopis sp. & \multirow{2}{*}{$\begin{array}{l}\text { Marchioni y Bellelli, } \\
\text { 2013; Pérez de Micou } \\
\text { et al., } 1992\end{array}$} \\
\hline & & 1 & aguja & $s / d$ & Prosopis sp. & \\
\hline Campo Nassif I & Holoceno tardío & 1 & aguja & $\mathrm{s} / \mathrm{d}$ & Schinus sp. & $\begin{array}{l}\text { Pérez de Micou et } \\
\text { al., } 2014\end{array}$ \\
\hline
\end{tabular}

Tabla 1. Antecedentes de instrumentos con morfología aguzada en Patagonia continental Argentina. En la columna tecnofactura las denominaciones son las asignadas por los autores de los trabajos citados.

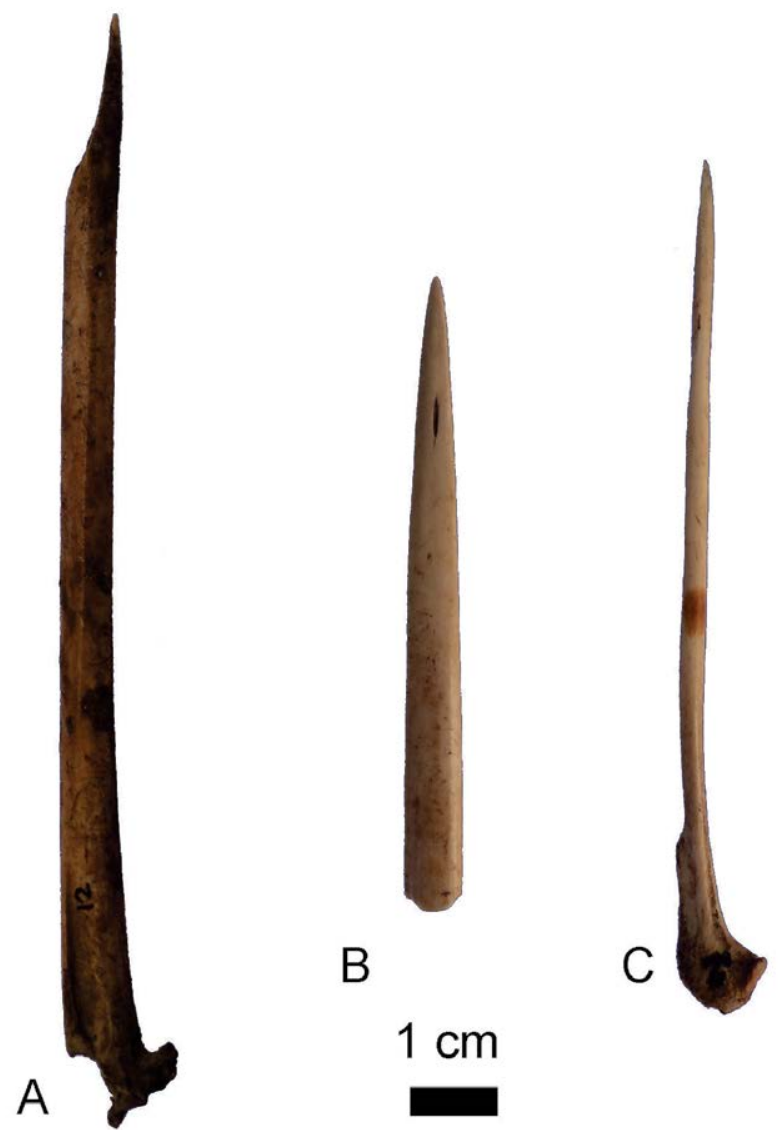

Figura 4. Punzones, agujas y leznas del sitio Cueva del Negro. A: punzón; $B$ : lezna o aguja; C: lezna o aguja (Beretta y Zubimendi, 2017). 
(petreles). Las dimensiones son 73, $26 \mathrm{~mm}$ de longitud máxima, 6,61-0,43 mm de ancho máximo a mínimo y una altura de 2,02-0,83 mm máxima a mínima (Figura 4B). La otra se realizó sobre la apófisis del pubis de un albatros con una longitud máxima de 92,8 $\mathrm{mm}, 2,89-0,97 \mathrm{~mm}$ de ancho máximo y mínimo y 2,08-0,82 $\mathrm{mm}$ de altura máxima y mínima (Figura 4C). Debido a la morfología de las piezas los autores consideran que pudieron ser empleados para trabajar materiales blandos como vegetales.

En este sitio además se recuperaron siete retocadores elaborados sobre hueso de guanaco (Beretta y Zubimendi, 2015), seis puntas de arpón monodentadas de espaldón simple pertenecientes a puntas de arpón móvil confeccionados sobre elementos óseos de cetáceos y pinnípedos (Beretta et al., 2013b) y dos ganchos de propulsor fabricados en hueso de pinnípedos (Beretta, Zubimendi, Castro y Ambrústolo, 2013a).

\section{Instrumentos en madera y hueso en el trabajo del cuero y la cestería}

Gusinde (1991) brinda una descripción de las acciones para la manufactura de los punzones en hueso a partir de observaciones entre los alakaluf de la costa chilena, sin embargo, no existe información similar sobre la realización de estos instrumentos en madera. Sobre los artefactos óseos, el autor señala que primero se extraía la carne de las extremidades de Phalacrocorax sp. (cormorán) y/o Diomedeidae (albatros). Luego, se realizaba una punta prolongada mientras se frotaban los huesos con una piedra áspera. El otro extremo, nudoso proporcionaba el mango apropiado para la mano (Gusinde, 1991, p. 286).

Con relación a la funcionalidad de estos instrumentos, Orquera y Piana (1999) mencionan el uso de punzones de madera y de hueso por parte de los yámanas a partir del análisis de fuentes etnográficas. Acerca de los punzones de madera, Hyades (1885, citado en Orquera y Piana, 1999) considera que eran empleados para la confección de canastos y Gusinde (1986) para la realización de orificios en corteza o cuero. En cuanto a los punzones de hueso, Orquera y Piana (1999) señalan el disenso entre distintos autores, de modo que para algunos se utilizaban para trenzar canastos y coser corteza, mientras que para otros los punzones de hueso eran usados sólo en la confección de canoas ya que para trenzar juncos se utilizarían los de madera (Hyades, 1885, citado en Orquera y Piana, 1999; Hyades y Deniker, 1891).

Para Fernández (1988-1990) los punzones de hueso podrían haber sido utilizados como alfileres, entendidos como elementos destinados a unir dos piezas de material flexible, dejando pasar la mayor parte del cuerpo del punzón y la epífisis conservada actuaría como tope. La aguja entonces, podría haberse utilizado posteriormente para pasar la tanza de costura a través del cuero (Beretta y Zubimeni, 2017).

Debido a que los punzones en soporte madera y hueso son asociados con la perforación y costura en cueros, corteza y entramado en cestería, se presentan a continuación los trabajos que han reconstruido las cadenas operativas de estas actividades para Patagonia. Gómez Otero (1996-1997) a partir de la observación de talleres de elaboración de quillangos con artesanos patagónicos menciona los siguientes pasos: cuereado, estaqueado, primer curtido, raspado, segundo curtido, sobado, corte, armado y costura, pintura y terminación. En el estaqueado, los artesanos mencionaron la utilización de astillas afiladas de arbustos. En el armado y costura se realiza la preparación de los tendones para obtener las fibras, muy finas pero fuertes, se humedecen y se enhebran con agujas, que en el caso de los talleres eran de acero (Gómez Otero, 1996-1997, p. 80). La autora describe que al ser cortos los tendones, se remachan las costuras, se unen los hilos y se cose borde contra borde, con puntadas cortas tipo anillado. También, aclara que antes de la adopción de agujas de metal, se agujereaban los cueros con punzones y luego por allí se introducían los hilos (Gómez Otero, 1996-1997). 
Por su parte, Marchione y Bellelli (2013) a partir de la secuencia de la preparación de quillangos descripta por Gómez Otero (1996-1997), fuentes etnohistóricas, entrevistas actuales y del registro arqueológico de Campo Moncada II, reconstruyen la cadena operativa del trabajo sobre cuero. En este sentido, el hallazgo de artefactos de cuero con costura, con orificios, algunos de ellos cuyos bordes presentan marcas de instrumentos cortantes así como nudos y cordeles, les permite diferenciar distintas etapas de trabajo en el registro arqueológico del sitio Campo Moncada II. Las autoras consideran que las varillas de Baccharis sp. L. con muescas en los extremos recuperadas en este sitio serían estacas y corresponderían al momento del estaqueado, tal como registraron en las fuentes etnohistóricas, etnográficas y entrevistas actuales. Las agujas de Prosopis sp. recuperadas en el sitio Campo Moncada II son asignadas a la etapa de armado y costura de la manta de cuero de acuerdo a las mismas fuentes que se describieron anteriormente (Marchione y Bellelli, 2013). Para las otras etapas no se menciona la incorporación de punzones o algún otro tipo de elemento vegetal. Por otra parte, Caviglia (2002) también analiza fuentes etnohistóricas, estudia los patrones de diseño de los mantos de cuero depositados en distintos museos y los vincula a su vez, con la cosmovisión del pueblo mapuche tehuelche. Además de mencionar varillas con punta de algarrobo y calafate, punzones y leznas de hueso, a través del relato de Schmid (1964) registra que los quillangos se mantenían cerrados con broches de madera, agujas o alfileres de bronce.

En el caso de la cestería, Gusinde (1986, p. 487) describió tres modalidades de cestería realizadas por los yámana. En todas las técnicas, las mujeres utilizaban continuamente "una varilla del largo de un dedo" terminada en punta o una lezna corta de hueso para realizar agujeros en el trenzado. A través de éstos se introducía los tallos de junco y se tiraba de los mismos para ajustarlos. En la Patagonia argentina, Pérez de Micou (1979-1982, 19881990, 1999, 2002, 2006) y Pérez de Micou y colaboradores (2014) estudiaron los materiales cesteros del registro arqueológico recuperado en el sitio Piedra Parada I, Cueva Chenque Haichol y Campo Moncada II. La autora identifica distintas técnicas de manufactura, tales como espiral, espiral cerrado de puntada simple, cestería plana y acordelado, aunque no menciona que en ellas intervenga algún artefacto en hueso o madera. A diferencia del procesamiento del cuero, Pérez de Micou (1999) señala tres aspectos importantes a considerar en el estudio de la cestería en el área de estudio: por un lado, las escasas evidencias de esta tecnología en el registro arqueológico, la pérdida de conocimiento sobre técnicas cesteras en la actualidad y el vacío de información histórica reciente. Esto último también fue observado por Ciampagna y Capparelli (2012) en el análisis de fuentes etnohistóricas en la región de Patagonia. Otro sitio en el que se recuperó cestería es en Cueva Galpón, Río Negro, donde se hallaron entramados de gramíneas asociados a un contexto enterratorio compuesto por una urdimbre de Piptochaetium sp. y/o Nassela cf. tenuissima unidos por una trama de Sporobolus rigens. Las mismas estaban atadas a varillas de Larrea cuneifolia. Todas las capas de entramado fueron confeccionadas con la técnica de acordelado simple abierto con torsión hacia la izquierda. También se recuperaron fragmentos de cordeles de distintos tipos laxos y compactos (Capparelli, Mange, Ciampagna y Prates, 2016).

En otras cadenas operativas, tales como la manufactura de cerámica, trabajos experimentales mencionan la utilización de espinas de Prosopis sp. para la decoración incisa de la pieza cuando ésta se encuentra todavía en un estado de plasticidad (Schuster, 2014.)

\section{Materiales y métodos}

\section{Material arqueobotánico}

Como se mencionó con anterioridad los instrumentos que se estudian en este trabajo forman parte de la colección arqueológica de los dueños de la Estancia El Amanecer, localidad arqueológica Punta Medanosa, en la provincia de Santa Cruz. Para el análisis 
morfológico externo de los dos instrumentos se tuvo en cuenta la forma y dimensiones de longitud máxima; ancho (máximo y mínimo), espesor (máximo y mínimo) en mm y se observaron bajo lupa binocular a fin de registrar la presencia de pulidos o rasgos de formatización. Para la identificación taxonómica de la madera se realizó el estudio anatómico de la madera a través de un microscopio de luz incidente Leica DM/LM. Se realizaron cortes delgados a mano alzada de las secciones de madera diagnósticos: transversal (CT), longitudinal tangencial (CLtg) y longitudinal radial (CLrd). Se montaron los cortes con gelatina glicerina y se observaron al microscopio. Los caracteres cualitativos microscópicos de diagnóstico se registraron usando la IAWA List of Microscope Features for Hardware Identification (Richter, Grosser, Heinz y Gasson, 2004; Wheeler, Baas y Gasson, 1989) y bibliografía específica (Ancibor y Pérez de Micou, 1995, 2002; Andreoni, 2014; Andreoni y Capparelli, 2012; Caruso Fermé, 2013; Ciampagna, 2015; Garibotti, 1998; Marconetto, 2002; Ortega y Marconetto, 2012; Roig y Videla, 2006-2010). Se realizó la comparación con cortes delgados del material de referencia moderno colectado y estudiado por la autora según la metodología que se describe a continuación.

\section{Material actual comparativo}

El material examinado corresponde a Schinus sp. L. Santa Cruz, procedente de Cañadón León, Caleta Olivia (ejemplar 90 de colección de herbario de Ciampagna, 2015) colectado y depositado en el Laboratorio de Arqueobotánica 129, División Arqueología, Museo de Ciencias Naturales de La Plata. Se hirvió un taco de este ejemplar de 1,5 cm de lado en agua y detergente industrial durante dos a cinco horas. Se realizaron luego los tres cortes histológicos diagnósticos (transversal, longitudinal tangencial y radial) con micrótomo a un espesor de $30 \mu \mathrm{m}$. Se decoloraron con hipoclorito de sodio al $50 \%$ durante cinco a diez minutos, luego se lavaron diez minutos aproximadamente sumergiéndolos en agua destilada. Posteriormente, se deshidrataron y tiñeron con safranina en una batería de alcoholes (alcohol $50^{\circ}$ con safranina al $1 \%$, alcohol 70 , alcohol $80^{\circ}$, alcohol $96^{\circ}$, alcohol $100^{\circ}$, alcohol y xilol en partes iguales y xilol $100 \%$ ). Se montaron los cortes en bálsamo de Canadá artificial y se describieron los caracteres cualitativos por medio de la observación al microscopio óptico de luz reflejada y siguiendo los criterios de Richter et al. (2004) y Wheeler et al. (1989).

\section{Resultados}

\section{Morfología externa}

Los dos instrumentos presentan un sector proximal con una forma protuberante y una sección distal que se afina hacia la punta. A ojo desnudo se observa que a medida que el instrumento se vuelve más angosto la superficie se encuentra pulida y se observa una textura lisa en la madera (ver Figura 2, sección distal de los instrumentos). Sin embargo, bajo lupa binocular no se registraron huellas tecnológicas sobre los artefactos, es decir líneas producidas por artefactos líticos o marcas de corte (Capparelli et al., 2009; Cueto, Capparelli, Ciampagna, Paunero y Castro, 2010). Al no haber una clasificación de los artefactos de madera y en función de las similitudes morfológicas y dimensiones con los instrumentos óseos de Cueva del Negro se define a estos objetos aquí estudiados como punzones (Tablas 1 y 2). Estas semejanzas se basan principalmente en la presencia de una protuberancia proximal en los instrumentos de madera que podría compararse con la epífisis proximal del hueso-soporte en los artefactos óseos. Estos últimos según los taxa pueden ser húmeros, tibiatarsos y en menor medida ulnas o radios. En los punzones óseos la diáfisis está fragmentada cerca de la epífisis distal y pulimentada para conformar una punta muy afilada. En el caso de los punzones de madera aquí estudiados se observa el aguzamiento siguiendo el plano tangencial de la madera y el pulido de la punta. No se observaron evidencias de decoración o grabados. 


\begin{tabular}{|l|c|c|}
\hline Medidas & Punzón 1 & Punzón 2 \\
\hline Longitud máxima & $106 \mathrm{~mm}$ & $150 \mathrm{~mm}$ \\
Acho máximo & $45 \mathrm{~mm}$ & $53 \mathrm{~mm}$ \\
Ancho sección media & $8 \mathrm{~mm}$ & $10 \mathrm{~mm}$ \\
Ancho mínimo & $1,5 \mathrm{~mm}$ & $1,3 \mathrm{~mm}$ \\
Espesor máximo & $29 \mathrm{~mm}$ & $34 \mathrm{~mm}$ \\
Espesor sección media & $7 \mathrm{~mm}$ & $13 \mathrm{~mm}$ \\
Espesor mínimo & $2 \mathrm{~mm}$ & $1 \mathrm{~mm}$ \\
\hline
\end{tabular}

Tabla 2. Medidas de los dos punzones analizados.

\section{Morfología interna: anatomía de la madera}

A partir del análisis anatómico de la madera se pudo determinar que los instrumentos se manufacturaron con leño de Schinus sp. L. (Fam. Anacardiaceae). Se describen, a continuación, los caracteres diagnósticos que permitieron la identificación taxonómica de la madera y que también fueron relevados para este género por otros autores.

Corte transversal. Se observaron anillos de crecimiento demarcados por la porosidad semicircular y una hilera de fibras achatadas. Los poros se disponen en bandas tangenciales en el leño temprano (Figura 5A), en patrón diagonal y radial (múltiples de cuatro, cinco y seis) en el leño tardío (Figura 5B). También se observaron poros solitarios y agrupados frecuentes en el leño tardío. Se registraron fibras de pared delgada a gruesa y parénquima axial ausente o extremadamente escaso.

Corte longitudinal tangencial. Los radios son uni a triseriados, heterogéneos, con extremos uniseriados de hasta cuatro células de alto (Figura 5C). Placas de perforación simples y oblicuas; se registra el espesamiento reticulado a lo largo del elemento de vaso (Figura 5D). Las punteaduras intervasculares son alternas poligonales (Figura 5E). Las fibras tienen punteaduras simples a punteaduras con rebordes diminutos. También se observaron espesamientos espiralados en tejido básico y fibras septadas presentes. Si bien el género se caracteriza por la presencia de canales radiales, éstos no se observaron en los cortes realizados.

Corte longitudinal radial. Se registraron punteaduras vasoradiales con rebordes muy reducidos hasta con apariencia simples. Las células del radio son procumbentes, cuadradas y erectas (de acuerdo con lo que observo en el CLRd del material de referencia y en el arqueológico también) (Figura 5F).

\section{Discusión}

En primer lugar, es importante destacar el hallazgo de estos punzones porque junto con el fragmento de punta de arpón de Berberis sp. son los únicos instrumentos manufacturados en madera de la CNSC. Cabe destacar su preservación tanto en Punta Medanosa y como en el sitio Cueva del Negro en el caso del fragmento de arpón. Es probable que los punzones de madera hayan estado poco tiempo expuestos debido a su buen estado de conservación. En el caso del fragmento de arpón hallado en estratigrafía fue la termoalteración de uno de los sectores del instrumento una de las variables preponderantes para su preservación (Capparelli et al., 2009; Ciampagna, 2015). Si 


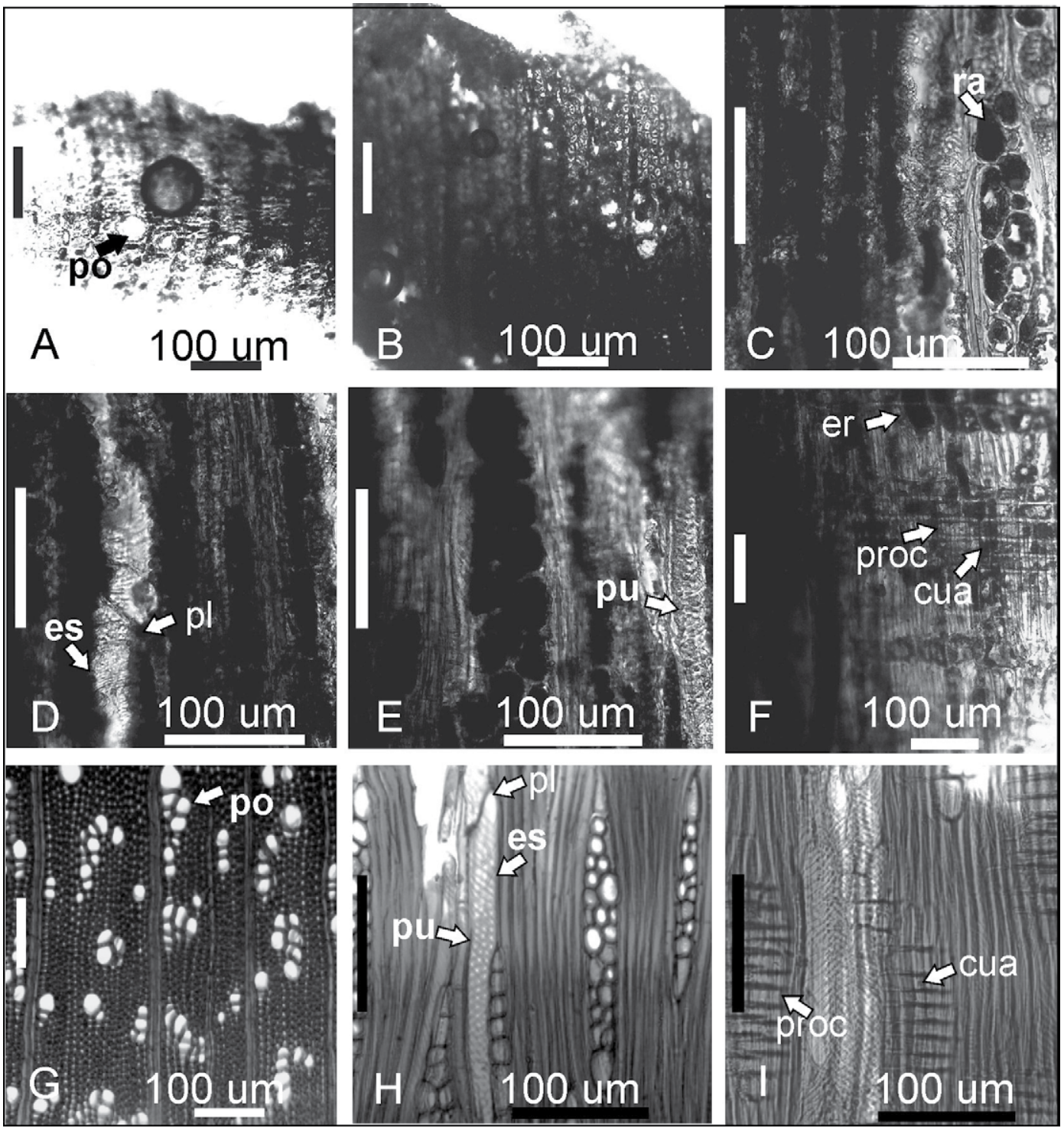

Figura 5. A-F) cortes delgados diagnósticos de los punzones de madera de Schinus sp. A) CT: poros en banda tangencial, B) CT: poros múltiples cortos radiales, parénquima axial ausente o escasa, C) CLtg: radios 1-3 seriados, D) CLtg: Espesamiento helicoidal en elemento de vaso, E) CLtg: punteaduras areoladas alternas y F) CLrd: células cuadradas del radio. G-I)

Material de referencia de Schinus sp. (Ciampagna 2015). G) CT: Se observan poros agrupados, múltiples cortos y largos, en disposición radial y diagonal, $H$ ) CLtg: Espesamiento reticulado en elementos de vasos, radios 1-3 seriados e I) CLrd: Células procumbentes, cuadradas y erectas.

bien se han registrado instrumentos de madera en sitios arqueológicos de Patagonia, en relación a otros elementos del registro arqueológico no son los que predominan.

En segundo lugar, el análisis morfológico y anatómico de los punzones hallados en el sector norte de la localidad de Punta Medanosa permiten discutir varios aspectos sobre manufactura y gestión de los recursos leñosos del área de estudio. El género Schinus presenta dos especies mencionadas para la provincia de Santa Cruz, una de ellas es $S$. johnstonii F.A. Barkley y la segunda es $S$. marchandii F. A. Barkley. (Zuloaga y Morrone, 1999). Por otra parte, en las provincias de Chubut, Mendoza, Neuquén y Río Negro, más precisamente en el ecotono entre el bosque y la estepa, se registra la especie S. patagonicus Phil. I. M. Johnst. (González et al., 2011; Zuloaga y Morrone, 1999). No fue posible obtener caracteres diagnósticos en los cortes realizados en los instrumentos que permitieran diferenciar estas especies, por lo tanto la identificación 
se realizó a nivel de género. Debido a que los instrumentos se hallaron en superficie se los asigna cronológicamente al Holoceno tardío, período en el que se considera que la vegetación no habría tenido cambios significativos (Ciampagna, 2015). En este sentido, es posible considerar que los artefactos se hayan manufacturado con materia prima vegetal del área, si bien como se mencionó anteriormente el género se distribuye con amplitud a lo largo de la estepa arbustiva actual.

En cuanto a las propiedades de la madera de Schinus sp. cabe señalar que se encuentra entre las maderas semiduras y semipesadas (Ciampagna, 2015, 2016). Además, aunque es necesario realizar estudios cuantitativos sobre el tamaño de fibras en ejemplares de la localidad, los datos obtenidos por Guerra y colaboradores (2012) dan cuenta de elementos cortos y por lo tanto de la resistencia de su madera. Estas propiedades pueden haber sido importantes en la selección del leño para la confección de estos instrumentos, que en el caso de los punzones óseos se caracterizan por producir penetración sin impacto (Tivoli, 2013).

Otro rasgo particular que presentan estos artefactos es la similitud que tienen con el punzón y agujas en hueso hallados tanto en el registro arqueológico de CNSC como en otras áreas de Patagonia (Beretta y Zubimendi, 2017; Morello et al., 2012; Scheinshon, Di Baja, Lanza y Tramaglino, 1993; Tivoli, 2013). En los punzones de madera aquí estudiados el extremo proximal más ancho presenta una protuberancia que podría haber servido como mango para el agarre, tal como lo describe Gusinde (1986) para los punzones de hueso, al tiempo que la sección distal se encuentra aguzada y pulida. En este sentido el sector proximal se asemejaría a la epífisis que puede permanecer en la confección de los punzones de hueso (Beretta y Zubimendi, 2017; Tivoli, 2013). Esta morfología no se observó en los punzones arqueológicos de madera que se han hallado en otros sitios arqueológicos de Patagonia, así como tampoco en las láminas ilustrativas de los etnógrafos que describieron los punzones de madera (Andreoni, 2014; Hyades 1885, citado en Orquera y Piana, 1999; Llano, 2010; Llano y Andreoni, 2012).

Es relevante la presencia de instrumentos confeccionados en ambos soportes, madera y hueso, de morfología similar en los sitios de CNSC. Esto indicaría una estrategia tecnológica en la que los grupos cazadores recolectores estarían combinando distintas materias primas para la realización de instrumentos similares (Beretta et al., 2013a; Capparelli et al., 2009; Ciampagna, 2015; Beretta y Zubimendi, 2017). Tanto en la localidad arqueológica de Punta Medanosa como en el sitio Cueva del Negro existe disponibilidad de materia prima ósea y vegetal. Es posible que la selección de materias primas pueda estar relacionada con una función diferente para el mismo tipo morfológico de artefacto. Este último es el caso del fragmento de arpón de madera y los cabezales de arpón en hueso hallados en el sitio Cueva del Negro. La madera de Berberis sp. con la se realizó el fragmento es semidura y de fibras cortas, propiedades que sumadas a la morfología del instrumento permiten sugerir que podría haber sido utilizado para presas chicas a diferencia de los cabezales de arpón óseos (Beretta et al., 2013a). En este sentido, se considera que existió una selección de las maderas en función de sus propiedades físico mecánicas (Capparelli et al., 2009; Ciampagna, 2015).

En relación a utilización de los punzones ya se ha mencionado su posible aplicación para la perforación y/o costura de cuero, cestería y el trenzado de fibras vegetales (Fernández, 1988-1990; Gusinde, 1986, 1991; Orquera y Piana, 1999; Tivoli, 2013; Zubimendi y Beretta, en prensa). En el caso de los punzones estudiados en este trabajo podemos inferir su aplicación para materiales blandos, posiblemente a modo de "alfileres" como menciona Fernández (1988-1990), broches de cierre para quillangos (Schmid, 1964), haciendo hincapié en que tienen un "tope". En este sentido aunque la madera de Schinus sp. sea de las más duras del área podría haber servido para la perforación de cuero, pero 
los punzones en hueso habrían sido más resistentes para la compresión. Es relevante la presencia de agujas de madera y espinas de Prosopis sp. y Schinus sp. en otros sitios de Patagonia continental Argentina (i.e. Campo Moncada II y Campo Nassif I) que se habrían utilizado en el estaqueado y la costura de los cueros (Marchione y Bellelli, 2013; Pérez de Micou et al., 2014). Se descarta que los punzones de madera aquí estudiados cumplieran la función de aguja ya que la protuberancia que presentan no permitiría que atraviese el cuero en su totalidad para una costura.

Con relación a la cestería, se observó que los materiales recuperados en distintos sitios arqueológicos de Patagonia continental Argentina fueron confeccionados mediante las técnicas utilizadas -espiral cerrado con costura simple, tejido plano, acordalado, acordalado abierto con torsión hacia la izquierda- de modo que son tejidos cerrados (Pérez de Micou, 1979-1982, 1988-1990,1999, 2002, 2006; Capparelli et al., 2016). En este tipo de cestería los punzones no podría pasar de forma completa debido a la protuberancia que presentan. Es posible entonces que los instrumentos analizados en este trabajo estuvieran relacionados con el trenzado de fibras vegetales, la incorporación de fibras en la trama y/o la separación de la urdimbre en la técnica en espiral abierto y menos probable su utilización en tejidos más cerrados. Asimismo, en este caso, los punzones de hueso señalados por Beretta y Zubimendi (2017) para el trabajo de materiales blandos podrían haber tenido una aplicación similar a los realizados en madera.

Por otro lado, también se pueden sugerir otros usos para estos punzones al considerar otros materiales recuperados en la localidad de Punta Medanosa, próximos al afloramiento de Roca del Cura tales como la cerámica. Es relevante el trabajo de Schuster (2014) quien utilizó espinas de Prosopis sp. para reproducir la decoración incisa de la cerámica y sugiere el uso eficaz de las mismas. En este sentido estos punzones podrían haber sido utilizados en la decoración de cerámica en el momento de plasticidad antes de su cocción.

\section{Conclusiones}

En este trabajo se analizaron dos punzones manufacturados con madera de Schinus sp. En la actualidad se observan arbustos de este taxa circundantes al área de hallazgo. Si bien existe más de una especie para este género en el área de estudio, se sugiere que existió una disponibilidad de materia prima vegetal para la manufactura local de los instrumentos así como ha sido postulado para el fragmento de arpón de Berberis sp. recuperado en el sitio Cueva del Negro (Beretta et al., 2013a; Capparelli et al., 2009; Ciampagna, 2015). Estos artefactos son los únicos instrumentos de madera recuperados hasta el momento en CNSC. A partir del análisis tecnomorfológico de los punzones de madera y su comparación con instrumentos en soporte óseo se sostiene la factibilidad de la Hipótesis 1 en la que se establece una similitud morfológica entre ambos. En este sentido se observa una mayor diversidad en las materias primas para la elaboración de instrumentos en el marco de una ocupación intensiva de la CNSC para el Holoceno tardío. En cuanto a la Hipótesis 2, la revisión sobre estudios de cadenas operativas en cuero, cestería y cerámica sugiere que los punzones de madera pudieron haber sido un elemento utilizado en algún momento para las tres. Sobre el trabajo en cuero podrían haber sido aplicados a modo de "alfileres", broches de cierre para quillangos y en menor medida para perforación de cuero ya que predominaría el trabajo sobre materiales blandos. En cestería podrían haber sido utilizados para la apertura de la urdimbre, el trenzado de fibras vegetales y la incorporación de las mismas en el tejido cestero. Se descartaría su utilización como aguja debido a la protuberancia proximal que presentan los instrumentos. En cuanto a la cerámica, podrían haber funcionado para la realización de decoración incisa de cerámica cuando ésta se encontraba en 
estado plástico antes de su cocción. Sin embargo, para abordar en mayor profundidad la segunda hipótesis y su contrastación se deberá realizar trabajos experimentales y abordar más exhaustivamente las propiedades mecánicas de la madera seleccionada. Por último, se considera relevante el análisis taxonómico de los instrumentos de madera y su asociación contextual en los casos en que esto es posible. Estos estudios permiten ampliar el conocimiento acerca de las estrategias tecnológicas no sólo de captura de animales, sino también, de elaboración de otros artefactos presentes en el registro arqueológico de Patagonia argentina.

\section{Agradecimientos}

A Luli y Sergio Vidal por permitir el análisis de estos artefactos y su cálida atención durante las campañas realizadas en la Estancia El Amanecer. A Heidi Hammond, Leandro Zilio y Pablo Ambrústolo por su colaboración en el acceso a los materiales de estudio. A Miguel Ángel Zubimendi por la revisión del manuscrito y a Aylen Capparelli por su constante ayuda. A Diego Gobbo por su colaboración con las imágenes. Al Lic. Orquera quien gentilmente brindó sus sugerencias y conocimiento sobre este tipo de artefactos. A los evaluadores, cuyas sugerencias contribuyeron a mejorar este trabajo. Estas investigaciones se desarrollaron con el apoyo financiero de CONICET, FONCyT y UNLP, proyectos dirigidos por la Dra. Aylen Capparelli, la Dra. Alicia Castro y el Dr. Miguel Ángel Zubimendi. 


\section{Q Referencias citadas}

"Ancibor, E. (1988-1990). Materiales leñosos: madera, caña y otros. Anales de Arqueología y Etnología, 43-45(2), 337-372.

"Ancibor, E. y Pérez de Micou, C. (1995). Identification of firewood species in the archaeological record of the Patagonian Steppe. Journal of Ethnobiology, 15(2), 189-200.

"Ancibor, E. y Pérez de Micou, C. (2002). Reconocimiento de especies vegetales combustibles en el registro arqueológico de la estepa patagónica. En C. Pérez de Micou (Ed.), Plantas y Cazadores en Patagonia (pp. 15-31). Buenos Aires: Facultad de Filosofía y Letras, Universidad de Buenos Aires.

"Andreoni, D. (2014). Plantas leñosas y estrategias humanas en el sur de Mendoza: una aproximación arqueobotánica. (Tesis Doctoral Inédita), Universidad Nacional de La Plata, Argentina.

》Andreoni, D. y Capparelli, A. (2012). El ser humano y la leña en la cordillera de Mendoza (Argentina) a lo largo del Holoceno: sitio arqueológico Arroyo Malo 3. Magallania, 40(1), 203-228.

"Beretta, M. y Zubimendi, M. A. (2015). ¿Qué sería de la industria lítica sin ellos?: Retocadores de hueso en la costa norte de Santa Cruz, Argentina. Atek Na, 5, 9-47.

" Beretta, M. y Zubimendi, M. A. (2017). El uso de huesos de aves como materia prima en la costa norte de Santa Cruz. Atek Na, 6, 9-49.

» Beretta, M., Zubimendi, M. A., Castro A. y Ambrústolo, P. (2013a). Ganchos de hueso en el sitio Cueva del Negro: evidencias de propulsores en la costa norte de Santa Cruz (Patagonia Argentina). Atek Na, 3, 9-34.

» Beretta, M., Zubimendi, M. A., Ciampagna, M. L., Ambrústolo, P. y Castro, A. (2013b). Puntas de arpón en la Costa Norte de Santa Cruz: primeros estudios de piezas recuperadas en estratigrafía en el sitio Cueva del Negro. Magallania, 41(1), 211-221.

"Capparelli A., Castro A. y Ciampagna, M. L. (2009). Descripción macroscópica e identificación anatómica de un instrumento de madera (¿arpón?) hallado en el sitio Cueva del Negro (costa norte de Santa Cruz, Argentina) En M. Salemme, F. Santiago, M. Álvarez, E. Piana, M. Vázquez y E. Mansur (Eds.), Arqueología de la Patagonia-Una Mirada desde el Último Confín (pp. 433-443). Ushuaia: Editorial Utopías.

"Capparelli, A., Mange, E., Ciampagna, M. L. y Prates L. (2016). Hunter gatherer archaeobotany of a mortuary context in Patagonia (Cueva Galpón, Argentina): artefactual, carpological, anthracological and other plant macroremains from ca. 3300 BP. Trabajo presentado en la $17^{\text {th }}$ Conference of the International Work Group for Paleoethnobotany, París, Francia.

"Caruso Fermé, L. (2013). Los Recursos Vegetales en Arqueología: Estrategias de Muestreo y Estudio del Material Leñoso. Buenos Aires: Dunken.

" Castro, A., Moreno, E., Andolfo, M. y Zubimendi, M. A. (2001). Distribución espacial de sitios en la Localidad de Punta Medanosa. Santa Cruz (Argentina). Relaciones de la Sociedad Argentina de Antropología, XXVI, 303-321.

»Castro, A., Zubimendi, M. A. y Ambrústolo, P. (2011). Archaeological littoral sites on the northern coast of Santa Cruz: valuable evidence of sea level changes on the continental Patagonian coasts (Argentina). Quaternary International, 245, 111-121. 
》 Caviglia, S. E. (2002). El arte de las mujeres Aónik'enk y Gününa Küna -Ka y Guaj'enk o Kay Gütruj (las capas pintadas). Relaciones de la Sociedad Argentina de Antropología, XXVII, 41-70.

»Ciampagna, M. L. (2015). Estudio de la interacción entre grupos cazadores recolectores de Patagonia y las plantas silvestres: el caso de la Costa Norte de Santa Cruz durante el Holoceno medio y tardío. (Tesis Doctoral Inédita), Universidad Nacional de La Plata, Argentina.

»Ciampagna, M. L. (2016). Prácticas en la gestión de recursos vegetales silvestres de grupos cazadores recolectores en los sitios Cormorán Quemado y Nido del Águila, Costa Norte de Santa Cruz, Patagonia Argentina. En F. Mena (Ed.), Arqueología de la Patagonia: De Mar a Mar (pp. 345-354). Santiago de Chile: Ediciones Ciep y Ñire Negro.

»Ciampagna, M. L. y Capparelli, A. (2012). Historia del uso de las plantas por parte de las poblaciones que habitaron la Patagonia continental Argentina. Cazadores-Recolectores del Cono Sur. Revista de Arqueología, 6, 45-75

"Cueto, M., Capparelli, A., Ciampagna, M. L., Paunero, M. y Castro, A. (2010). Prácticas postcolecta y material leñoso: análisis de residuos y huellas microscópicas de origen vegetal, sobre artefactos de roca tallada, utilizados en contextos experimentales. En R .Bárcena y H. Chiavazza (Eds.), Arqueología Argentina en el Bicentenario de la Revolución de Mayo, Actas del XVII Congreso Nacional de Arqueología Argentina (pp. 12051210). Mendoza: Instituto de Ciencias Humanas Sociales y Ambientales (INCIHUSA), CONICET.

»De Porras, M. E. (2010). Dinámica de la vegetación de la Meseta Central de Santa Cruz durante los últimos 11.000 años: forzantes bióticos y abióticos. (Tesis Doctoral Inédita), Universidad Nacional de Mar del Plata, Argentina.

"Fernández J. (1988-1990). El Instrumental de hueso. Anales de Arqueología y Etnología 43-45(1), 243-286.

»Garibotti, I. A. (1998). Análisis de la estructura anatómica de carbones arqueológicos de sitios incaicos (ca. 1480-1530 d.C.) del valle de Uspallata (Mendoza, Argentina). Boletín de la Sociedad Argentina de Botánica, 33(3-4), 195-205.

» Gómez Otero, J. (1996-1997). Rescate y reinserción de los mantos de pieles (quillangos) indígenas de Patagonia en la Provincia del Chubut (Argentina). Cuadernos del Instituto Nacional de Antropología y Pensamiento Latinoamericano, 17, 69-87.

» González, S. B., Guerra, P. E., Van Baren, C. M., Di Leo Lira, P. y Bandoni, A. L. (2011). El aceite esencial de tallos y hojas de Schinus patagonicus (Phil.) Johnst. en el ecotono de la Patagonia, Argentina. Dominguezia, 27(1), 33-39.

» Guerra, P., González, S., Kirner, H., Retta, D., Di Leo Lira, P. y Gómez, M. (2012). Aspectos anatómicos del leño y composición de los aceites esenciales de especies arbustivasleñosas del ecotono y la estepa del noroeste de la Provincia de Chubut. Dominguezia, 28(1), 13-44

» Gusinde, M. (1986). Los indios de Tierra del Fuego. Los Yámana (Tomo II, Volumen I). Buenos Aires: Centro Argentino de Etnología Americana.

"Gusinde, M. (1991). Los indios de Tierra del Fuego, Los Halakwulup (Tomo III, Volumen I). Buenos Aires: Centro Argentino de Etnología Americana.

" Hammond,H.(2013). Propuesta metodológica para el estudio de conjuntos malacológicos de sitios concheros: su aplicación en la costa norte de Santa Cruz (Patagonia Argentina). La Zaranda de Ideas. Revista de Jóvenes Investigadores en Arqueología, 9(2), 77-102.

» Hammond, H., Zilio, L. y Zubimendi, M. A. (2016) Proyecto Arqueología de la Costa Norte de Santa Cruz. Informe de las Actividades de campo remitido a la Provincia de Santa Cruz. Manuscrito inédito. 
» Hammond, H., Zubimendi, M. A. y Zilio, L. (2013). Composición de concheros y uso del espacio: aproximaciones al paisaje arqueológico costero en Punta Medanosa. Anuario de Arqueología, 5, 67-84.

» Hyades, P. y Deniker, J. (1891). Mission Scientifique du Cap Horn 1882-1883 (Tomo 7). París: Anthropologie Etnhographie, Gauthiers-Villars.

»Llano, C. (2010). Aprovechamiento de los recursos vegetales entre las sociedades cazadorasrecolectoras del sur de Mendoza. (Tesis Doctoral Inédita), Universidad Nacional del Comahue, Argentina.

» Llano, C. y Andreoni, D. (2012). Caracterización espacial y temporal en el uso de los recursos vegetales entre los grupos cazadores recolectores del sur mendocino durante el Holoceno. En A. Gil y G. Neme (Eds.), Paleoecología Humana en el Sur de Mendoza: Perspectivas Arqueológicas (pp. 57-84). Buenos Aires: Sociedad Argentina de Antropología.

» Marchione, P. C. y Bellelli, C. (2013). El trabajo del cuero entre los cazadores recolectores de la Patagonia centro-septentrional. Campo Moncada 2 (Valle medio del río Chubut). Relaciones de la Sociedad Argentina de Antropología, XXXVIII(1), 223-246.

» Marconetto, B. (2002). Análisis de los vestigios de combustión de los sitios Alero Don Santiago y Campo Moncada. En C. Pérez de Micou (Ed.), Plantas y Cazadores en Patagonia (pp. 33-51). Buenos Aires: Facultad de Filosofía y Letras, Universidad de Buenos Aires.

» Morello, F., Torres, J., Martínez, I., Rodríguez, K., Arroyo Kalin, M., French, C., Sierpe, V. y San Román, M. (2012). Arqueología de la Punta Santa Ana: reconstrucción de secuencias de ocupación de cazadores recolectores marinos del Estrecho de Magallanes, Patagonia Austral, Chile. Magallania, 40(2), 129-149.

» Oliva, G., González, L. y Rial, P. (2001). El ambiente en Patagonia austral. En P. Borrelli y G. Oliva (Eds.), Ganadería Sustentable en la Patagonia Austral (pp. 17-8o). Buenos Aires: Centro Regional Patagonia Sur INTA, Estación Experimental Agropecuaria Santa Cruz.

»Orquera, L. y Piana, E. (1999). La Vida Material y Social de los Yámana. Buenos Aires: EUdeBA.

» Ortega, F. y Marconetto, B. (2012). La explotación de recursos combustibles: su uso y representación en la costa rionegrina a través de los restos antracológicos. En F. Borella y M. Cardillo (Ed.), Arqueología de Pescadores y Marisqueadores en Nordpatagonia, Descifrando un Registro de Más de 6000 años (pp. 111-127). Buenos Aires: Editorial Dunken.

» Pérez de Micou, C. (1979-1982). Sitio Piedra Parada (PP1) Departamento Languiñeo, Provincia de Chubut. Cuadernos del Instituto Nacional de Antropología y Pensamiento Latinoamericano, 9, 97-112.

» Pérez de Micou, C. (1988-1990). Estudio de una pieza cestera. Anales de Arqueología y Etnología, 43-45(2), 327-330.

»Pérez de Micou, C. (1999). La recolección vegetal y la paleoetnobotánica en Patagonia y Puna. En C. Aschero, M. L. Korstanje y P. Vuoto (Eds.), En los Tres Reinos: Prácticas de Recolección en el Cono Sur de América (pp. 121-128). San Miguel de Tucumán: Instituto de Arqueología y Museo, Facultad de Ciencias Naturales e Instituto Miguel Lillo, Universidad Nacional de Tucumán.

»Pérez de Micou, C. (2002). Tecnología cestera en Patagonia fechando artefactos. En C. Pérez de Micou (Ed.), Plantas y Cazadores en Patagonia (pp. 55-63). Buenos Aires: Facultad de Filosofía y Letras, Universidad de Buenos Aires.

»Pérez de Micou, C. (Ed.). (2006). El Modo de Hacer las Cosas, Atefactos y Ecofactos en Arqueología. Buenos Aires: Facultad de Filosofía y Letras, Universidad de Buenos Aires. 
»Pérez de Micou, C., Bellelli C. y Aschero, C. (1992). Vestigios minerales y vegetales en la determinación del territorio de explotación de un sitio. En J. Lanata y L. Borrero (Eds.), Análisis Espacial en la Arqueología Patagónica (pp. 53-82). Buenos Aires: Ediciones Búsqueda de Ayllu.

»Pérez de Micou, C., López Campeny, S. M. L. y Lessa Costa, R. (2014). Basketry of South America. En H. Selin (Ed.), Encyclopaedia of the History of Science, Technology, and Medicine in Non-Western Cultures (pp. 1-22). Dordrecht: Springer Science and Business Media.

» Richter, H., Grosser, D., Heinz, I. y Gasson, P. (2004). IAWA List of microscopic features for softwood identification. IAWA Journal, 25(1), 1-70.

» Roig, F. A. y Videla, E. (2006-2010). Anatomía de la madera de arbustos de montaña del NW de Mendoza, Argentina. Xama, 19-23, 157-238.

» Scheinsohn, V., Di Baja, A., Lanza, M. y Tramaglino, L. (1993). El aprovechamiento de la avifauna como fuente de materia prima ósea en la Isla Grande de Tierra del Fuego: Lancha Packewaia; Shamakush y Túnel I. Arqueología, 2, 135-148.

» Schiavini, A., Yorio, P., Gadini, P. A., Raja Rey, A. y De Boerma, P. (2005). Los pingüinos de las costas argentinas: estado poblacional y conservación. Hornero, 20(1), 5-23.

»Schmid, T. (1964). Misionando por Patagonia Austral 1858-1865, Usos y Costumbres de los Indios Patagones. Buenos Aires: Academia Nacional de la Historia.

»Schuster, V. (2014). La organización tecnológica de la cerámica de cazadores recolectores. Costa Norte de la Provincia de Chubut (Patagonia Argentina). Relaciones de la Sociedad Argentina de Antropología, XXXIX(1), 203-231.

» Tivoli, A. M. (2013). Aprovechamiento de materias primas óseas de aves para la confección de punzones huecos en la región de canal Beagle. Intersecciones en Antropología, 14, 251262.

»Wheeler, E., Baas, P. y Gasson, P. (1989). List of microscopic features for hardwood identification with an Appendix on non-anatomical information. IAWA Bulletin, 10(3), 219-332.

»Zubimendi, M. A., Ambrústolo, P., Beretta, M., Mazzitelli, L., Hammond, H., Zilio, L., Ciampagna, M. L., Trola, V., Plischuck, M. y Castro, A. (2011). Sitio Cueva del Negro: un caso de aprovechamiento intensivo de los recursos marinos en la costa norte de Santa Cruz (Patagonia Argentina). Revista de Estudios Marítimos y Sociales, 4, 51-59.

»Zubimendi, M. A., Ambrústolo, P., Zilio, L. y Castro A. (2015). Continuity and discontinuity in the human use of the north coast of Santa Cruz (Patagonia, Argentina) through its radiocarbon record. Quaternary International, 356, 127-146.

»Zuloaga F. y Morrone O. (1999). Catálogo de las Plantas Vasculares de la República Argentina (Volumen II). Saint Louis: Monographs in Systematic Botany, Missouri Botanical Garden. http://www.darwinion.gov.ar (Acceso: 27 de Julio, 2017). 